LISA Pathfinder data analysis

This article has been downloaded from IOPscience. Please scroll down to see the full text article.

2011 Class. Quantum Grav. 28094006

(http://iopscience.iop.org/0264-9381/28/9/094006)

View the table of contents for this issue, or go to the journal homepage for more

Download details:

IP Address: 194.94.224.254

The article was downloaded on 16/01/2012 at 13:30

Please note that terms and conditions apply. 


\title{
LISA Pathfinder data analysis
}

\author{
F Antonucci ${ }^{1}$, M Armano $^{2}$, H Audley $^{3}$, G Auger $^{4}$, M Benedetti $^{5}$, \\ P Binetruy $^{4}, \mathbf{C}$ Boatella $^{6}$, J Bogenstahl $^{3}$, D Bortoluzzi ${ }^{7}$, P Bosetti $^{7}$, \\ M Caleno $^{8}$, A Cavalleri $^{1}$, M Cesa $^{8}$, M Chmeissani $^{9}, \mathbf{G ~ C i a n i ~}^{10}$, \\ A Conchillo ${ }^{11}, \mathrm{G}_{\text {Congedo }}{ }^{1}$, I Cristofolini $^{7}, \mathbf{M}$ Cruise $^{12}, \mathrm{~K}^{1}$ Danzmann $^{3}$, \\ F De Marchi ${ }^{1}$, M Diaz-Aguilo $^{13}$, I Diepholz $^{3}$, G Dixon $^{12}$, R Dolesi $^{1}$, \\ J Fauste $^{2}$, L Ferraioli ${ }^{1}$, D Fertin ${ }^{8}$, W Fichter ${ }^{14}$, E Fitzsimons ${ }^{15}$, \\ M Freschi $^{2}$, A García Marin ${ }^{3}$, C García Marirrodriga ${ }^{8}$, L Gesa $^{11}$, \\ D Giardini $^{16}$, C Grimani $^{17}$, A Grynagier ${ }^{14}$, B Guillaume ${ }^{8}$, F Guzmán $^{18}$,

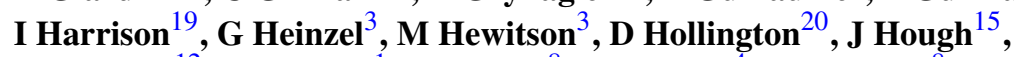 \\ D Hoyland $^{12}$, M Hueller ${ }^{1}$, J Huesler ${ }^{8}$, O Jeannin ${ }^{4}$, O Jennrich ${ }^{8}$,

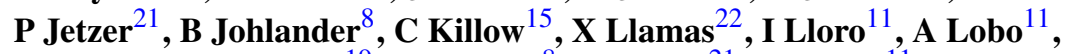 \\ R Maarschalkerweerd ${ }^{19}, \mathbf{S ~ M a d d e n ~}^{8}$, D Mance ${ }^{21}$, I Mateos ${ }^{11}$, \\ P W McNamara ${ }^{8}$, J Mendes ${ }^{19}$, E Mitchell ${ }^{20}$, A Monsky ${ }^{3}$, D Nicolini ${ }^{8}$,
} D Nicolodi ${ }^{1}$, M Nofrarias $^{3}$, F Pedersen $^{8}$, M Perreur-Lloyd $^{15}{ }^{1}$ A Perreca ${ }^{1}$,

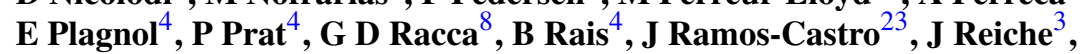
J A Romera Perez ${ }^{8}$, D Robertson ${ }^{15}$, H Rozemeijer ${ }^{8}$, J Sanjuan ${ }^{10}$, M Schulte $^{20}$, D Shaul ${ }^{20}$, L Stagnaro ${ }^{8}$, S Strandmoe ${ }^{8}$, F Steier $^{3}$, T J Sumner ${ }^{20}$, A Taylor ${ }^{15}$, D Texier ${ }^{2}$, C Trenkel ${ }^{24}$, D Tombolato ${ }^{1}$,

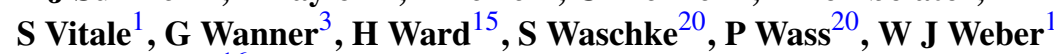
and $\mathbf{P}$ Zweifel $^{16}$

\footnotetext{
${ }^{1}$ Dipartimento di Fisica, Università di Trento and INFN, Gruppo Collegato di Trento, 38123 Povo, Trento, Italy

${ }^{2}$ European Space Astronomy Centre, European Space Agency, Villanueva de la Cañada, 28692 Madrid, Spain

${ }^{3}$ Albert-Einstein-Institut, Max-Planck-Institut für Gravitationsphysik und Universität Hannover, 30167 Hannover, Germany

${ }^{4}$ APC UMR7164, Université Paris Diderot, Paris, France

5 Dipartimento di Ingegneria dei Materiali e Tecnologie Industriali, Università di Trento and INFN, Gruppo Collegato di Trento, Mesiano, Trento, Italy

${ }^{6}$ CNES, DCT/AQ/EC, 18 Avenue Edouard Belin, 31401 Toulouse, Cédex9, France

${ }^{7}$ Dipartimento di Ingegneria Meccanica e Strutturale, Università di Trento and INFN, Gruppo Collegato di Trento, Mesiano, Trento, Italy

${ }^{8}$ European Space Technology Centre, European Space Agency, Keplerlaan 1, 2200 AG

Noordwijk, The Netherlands

${ }^{9}$ IFAE, Universitat Autònoma de Barcelona, E-08193 Bellaterra (Barcelona), Spain

${ }^{10}$ Department of Physics, University of Florida, Gainesville, FL 32611-8440, USA

11 ICE-CSIC/IEEC, Facultat de Ciències, E-08193 Bellaterra (Barcelona), Spain

12 Department of Physics and Astronomy, University of Birmingham, Birmingham, UK

13 UPC/IEEC, EPSC, Esteve Terrades 5, E-08860 Castelldefels, Barcelona, Spain

14 Institut für Flugmechanik und Flugregelung, 70569 Stuttgart, Germany

15 Department of Physics and Astronomy, University of Glasgow, Glasgow, UK

${ }^{16}$ Institut für Geophysik, ETH Zürich, Sonneggstrasse 5, CH-8092, Zürich, Switzerland

${ }^{17}$ Istituto di Fisica, Università degli Studi di Urbino/INFN Urbino (PU), Italy

18 NASA - Goddard Space Flight Centre, Greenbelt, MD 20771, USA

${ }^{19}$ European Space Operations Centre, European Space Agency, 64293 Darmstadt, Germany
} 
${ }^{20}$ The Blackett Laboratory, Imperial College London, UK

${ }^{21}$ Institut für Theoretische Physik, Universität Zürich, Winterthurerstrasse 190, CH-8057 Zürich, Switzerland

${ }^{22}$ NTE-SENER, Can Malé, E-08186, Lliçà d'Amunt, Barcelona, Spain

${ }^{23}$ Universitat Politècnica de Catalunya, Enginyeria Electrònica, Jordi Girona 1-3, 08034

Barcelona, Spain

${ }^{24}$ Astrium Ltd, Gunnels Wood Road, Stevenage, Hertfordshire, SG1 2AS, UK

E-mail: martin.hewitson@aei.mpg.de

Received 5 October 2010, in final form 22 December 2010

Published 18 April 2011

Online at stacks.iop.org/CQG/28/094006

\begin{abstract}
As the launch of LISA Pathfinder (LPF) draws near, more and more effort is being put in to the preparation of the data analysis activities that will be carried out during the mission operations. The operations phase of the mission will be composed of a series of experiments that will be carried out on the satellite. These experiments will be directed and analysed by the data analysis team, which is part of the operations team. The operations phase will last about 90 days, during which time the data analysis team aims to fully characterize the LPF, and in particular, its core instrument the LISA Technology Package. By analysing the various couplings present in the system, the different noise sources that will disturb the system, and through the identification of the key physical parameters of the system, a detailed noise budget of the instrument will be constructed that will allow the performance of the different subsystems to be assessed and projected towards LISA. This paper describes the various aspects of the full data analysis chain that are needed to successfully characterize the LPF and build up the noise budget during mission operations.
\end{abstract}

PACS numbers: $95.55 . \mathrm{Ym}, 04.80 . \mathrm{Nn}$

(Some figures in this article are in colour only in the electronic version)

\title{
1. Introduction
}

The LISA space-borne Gravitational Wave Observatory [1], when operational, will provide a unique view of the universe at spectral frequencies unobservable with ground-based detectors. As well as directly observing the gravitational radiation from a number of known sources, LISA will also provide the opportunity to observe unexpected signals and sources.

In order to detect the known sources with a high signal-to-noise ratio, the strain sensitivity of LISA has to be of the order of $10^{-21} \mathrm{~Hz}^{-1 / 2}$ at milliHertz frequencies. Many of the technologies required to build an instrument that can achieve such a strain sensitivity are currently being constructed and will be tested on the LISA Pathfinder satellite (LPF). In order to reduce the cost and complexity of the LPF, the performance of the various subsystems required to achieve the desired sensitivity for LISA has been relaxed such that the main goal for the LPF is to demonstrate the ability to put a test particle in free-fall to such a level that the residual external force per unit mass acting on the particle is below $3 \times 10^{-14} \mathrm{~m} \mathrm{~s}^{-2} \mathrm{~Hz}^{-1 / 2}$ at 
$1 \mathrm{mHz}$. Additionally, in order to show that the performance of the various subsystems under test is good enough, or can be extrapolated to a level suitable for LISA, the final measured performance of the LPF will need to be completely explained in the measurement bandwidth ( $1 \mathrm{mHz}$ through $30 \mathrm{mHz}$ ). This means that, as well as assessing the residual forces acting on the test particle, one of the key data analysis activities will be to build up as complete a noise model as possible.

In more concrete terms, the LPF will place a macroscopic test particle, a $2 \mathrm{~kg}$ testmass (TM) made from a gold-platinum alloy, in free-fall. In order to assess the residual acceleration of the test-mass, a second, nominally identical, test-mass is flown and a differential measurement is made. This allows the relatively noisy jitter of the spacecraft (SC) to be isolated from the measurement process. From the differential measurement, we then estimate the residual differential acceleration of the two bodies [2]. A SC is required to shield the two TMs from external influences (such as solar radiation pressure) and to provide a platform for the measurement equipment. To achieve the best possible free-fall, the forces acting on the first TM along the $x$-axis (the axis joining the centres of mass of the two test-masses) will be kept to a minimum. As such, no control forces will be directly applied to that TM. This leads to a control scheme where the jitter of the SC relative to the first TM is measured and minimized via a drag-free [3] control loop utilizing micro-Newton thrusters attached to the SC. The differential position of the two TMs is also controlled via the electrostatic actuators surrounding the second TM. The other degrees of freedom of the three bodies are also controlled via a mixture of the electrostatic actuators surrounding the two TMs and the SC thrusters. The full control scheme is referred to as the drag-free attitude and control system (DFACS) [4].

All together, LPF is a complicated system of nested and coupled control loops operating across 15 degrees of freedom. In order to achieve the best possible level of free-fall and to establish a complete noise budget for the $x$-axis (as described above), these various loops and couplings have to be characterized and optimized through a series of dedicated experiments.

The TMs, sensors and actuators, together with supporting diagnostic and computer systems, make up the LISA Technology Package (LTP), the core instrument on-board LISA Pathfinder. The LTP contains two main sensor systems which can be used to readout the different degrees of freedom of the two TMs relative to each other and to the SC. The gravitational reference system (GRS) [5] is based on a pattern of electrodes surrounding both TMs and capacitively reads the SC-TM relative motion along all 6 degrees of freedom to within $1 \mathrm{~nm} \mathrm{~Hz} z^{-1 / 2}$. The TMs are polarized via an oscillating electric field to allow their position to be capacitively readout. By simultaneously applying voltages at different frequencies to the same electrodes it is also possible to electrostatically apply forces and torques to reposition and rotate the TMs themselves according to the commanded forces and torques coming from the DFACS controllers.

The second, and more sensitive, sensor system is interferometric and provides readouts of the $x$-axis position of the first TM relative to the SC (the $X_{1}$ interferometer) and the differential position of the two TMs (the $X_{12}$ interferometer) to an accuracy of around $9 \mathrm{pm} \mathrm{Hz}$ at $1 \mathrm{mHz}$. In addition, two interferometric angular readouts of each TM via differential wavefront sensing are implemented; these are accurate to about $20 \mathrm{nrad} \mathrm{Hz}^{-1 / 2}$. The laser, modulators, optical components, phase-meter, and processing computer together form the optical metrology subsystem (OMS) [6].

These two sensors (the GRS and the OMS) allow for two main science control modes. The difference between the two is in how the $x$-position of the SC relative to the first TM is measured: the first (Science Mode 1) uses the capacitive sensor; the second (Science Mode 1 all-optical) uses the output of the $X_{1}$ interferometer. In both modes, the position of the second 


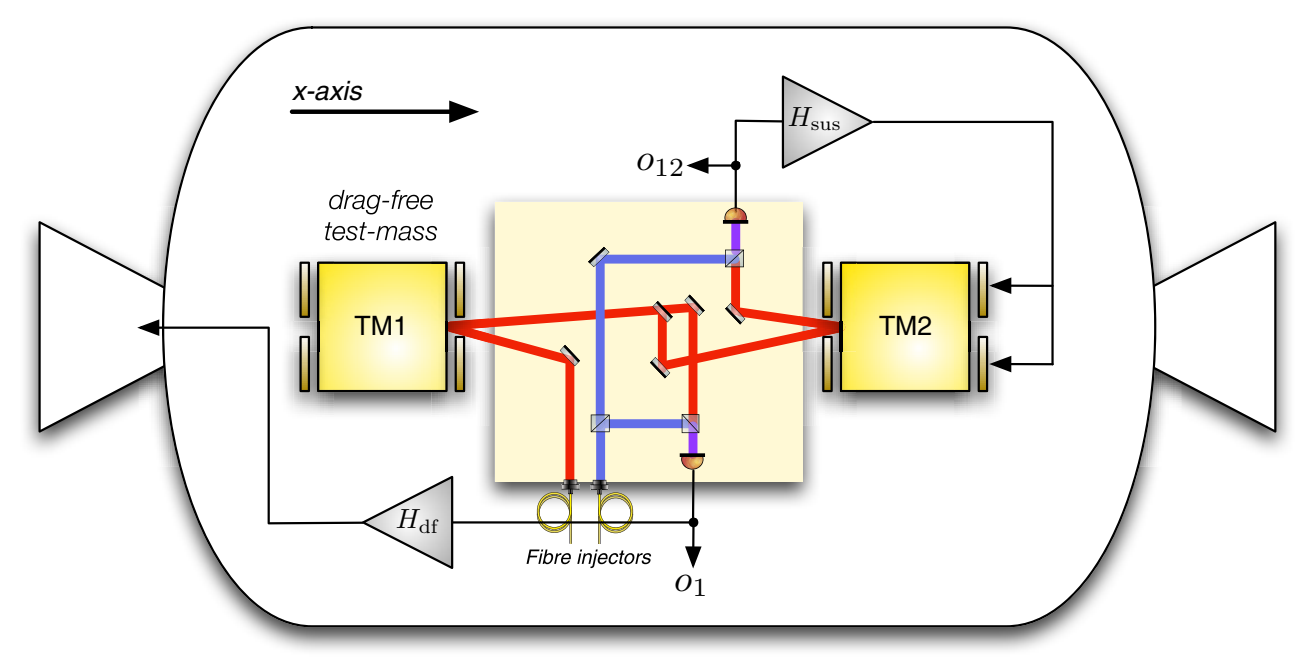

Figure 1. Schematic representation of the $x$-axis control of the LPF in control mode Science Mode 1 all-optical. Here, the first test-mass, TM1, is drag-free, and the second test-mass, TM2, follows TM1. The two interferometer readouts, $o_{1}$ and $o_{12}$ are indicated. In practice, all 12 micro-Newton thrusters are used when moving the SC along $x$ according to the output of the drag-free controller, $H_{\mathrm{df}}$, and both pairs of electrodes are used to actuate the second test-mass along $x$ according to the output of the low-frequency suspension controller, $H_{\text {sus. }}$.

TM relative to the first is controlled using the output of the $X_{12}$ interferometer. Further details of the main science objectives of the LPF can be found in this volume in [7] and a schematic of the LPF in Science Mode 1 all-optical is shown in figure 1.

The LPF is a short duration mission, where the LTP phase lasts about 90 days. During that time, the full optimization and characterization of all the subsystems must take place. In order to do that the various experiments that will be performed need to be analysed in real time so that following experiments can be adjusted and/or rescheduled to allow optimal use of the available mission time. For example, the available actuators (micro-Newton thrusters and electrostatic actuators) will need to be balanced and diagonalized in some of the early experiments, thus suppressing various noise sources. In addition, an experiment may reveal that some part of the system is not operating correctly, and should be switched off or optimized to reduce its noise contribution. Similarly, the identification of particular parameters, like actuator gains or coupling coefficients, may require the stimulus signals in subsequent experiments to be reduced or increased in order to maintain sufficient signal-to-noise ratio, or to not exceed limits of the system. For more discussion, see section 3 and [7, 8].

To ensure that it is possible to gain the maximum science return from the mission, the various experiments needed to characterize the instrument will be planned in advance and packed together in a preliminary mission timeline. Being able to analyse the experiments in real time implies that the data analysis for each experiment needs to be planned, prepared and tested in advance of the mission. Additionally, to ensure that we design the optimal set of experiments given the information to date, we need to simulate and validate each experiment prior to the launch. The design, simulation, and analysis of the experiments and data analysis pipelines, together with the supporting computing infrastructure [9], are the main tasks of the LTP data analysis team. The rest of this paper aims to provide an overview of the activities 


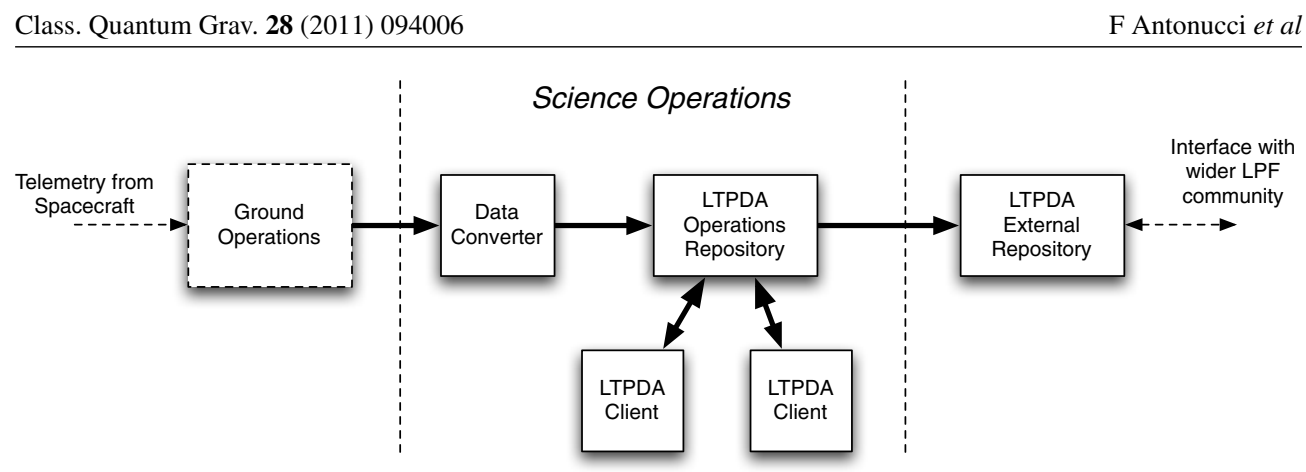

Figure 2. Elements comprising the data flow chain for the mission science operations. The software components being developed as part of the LTP data analysis activity are depicted by the solid boxes.

and status of each of those tasks. We also aim to provide references, when appropriate, to the more detailed analyses that are being developed to allow for a more in-depth off-line treatment of the experiment data.

\section{Data analysis infrastructure}

In order to carry out the quasi-on-line data analysis required to make real-time decisions about the planned experiments, a robust and complete data analysis chain was designed and built. The main components of that data flow chain are shown in figure 2.

Data are received from the SC at an ESA ground station in the form of telemetry packets. These packets are then unpacked and processed to provide individual data streams for each of the thousands of recorded signals from the SC. These data streams are then delivered to the science operations team where they are converted in to the format supported by the data analysis tools and stored in a local data repository. From there, the data can be analysed by multiple scientists, and any analysis results placed back in the data repository.

The data analysis tools are packaged in the form of a MATLAB ${ }^{\odot}$ toolbox which implements an object-oriented data analysis infrastructure based around the concept of analysis objects (AOs). One of the key requirements of this data analysis system is that any results which are produced should be able to be interpreted at a later stage without relying on additional documentation or knowledge. As such, AOs can track what happens to themselves as they are passed through a data analysis pipeline. This means that each algorithm that is implemented in the toolbox attaches some information to a history tree which is stored inside the output AOs. By doing this, any analysis result can be inspected to reveal a full processing history all the way back to the original raw data coming from the satellite. Each node in the history tree contains full details of the algorithm used, the version of the code, the values of all configuration parameters used, and details of the input objects. Full details of the data analysis toolbox and its design can be found in [9].

\section{The experiment master plan}

As previously stated, LISA Pathfinder aims to demonstrate the technological readiness of LISA by showing a level of free-fall that can reasonably be extrapolated to the required LISA performance. In order to do the extrapolation, it is essential that, in addition to reaching the 


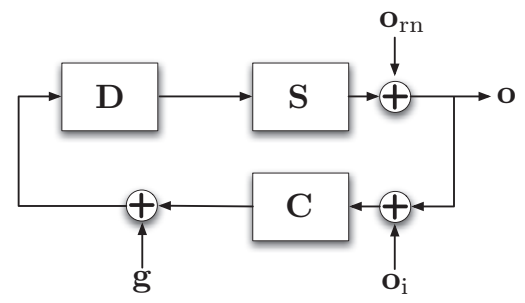

Figure 3. Schematic representation of the $x$-axis system model. The interferometer is represented by the sensing matrix, $\mathbf{S}$, and has a vector of output signals, $\mathbf{o}$. The DFACS controllers are represented by the matrix $\mathbf{C}$. The actuation and resulting dynamics are represented by $\mathbf{A}$ and $\mathbf{D}$, respectively. We show three inputs to the system: readout or sensing noise, $\mathbf{o}_{\text {rn }}$; guidance input signals, $\mathbf{o}_{i}$; and external force inputs, $\mathbf{g}$.

free-fall goal, the measurement of the differential test-mass acceleration can be fully explained in terms of its individual noise contributions. Once a full noise budget is built up, the noise performance of each of the subsystems can then be individually extrapolated towards LISA. The observed noise of each subsystem is sometimes best characterized in terms of residual accelerations (or forces), and sometimes in terms of displacements of the test-masses. In either case, a detailed and accurate model of the entire system must be developed throughout the mission.

The main structure of this dynamical model along the $x$-axis can be expressed as

$$
\mathbf{o}=\frac{\mathbf{o}_{\mathrm{rn}}}{1+\mathbf{G}}+\frac{\mathbf{G o}_{\mathrm{i}}}{1+\mathbf{G}}+\frac{\mathbf{S D g}}{1+\mathbf{G}}
$$

where $\mathbf{G}$ is the open-loop gain of the system composed of

$$
\mathbf{G}=\mathbf{C D S}
$$

where $\mathbf{D}$ is the dynamical response matrix of the system, $\mathbf{S}$ is the sensing matrix of the system, and $\mathbf{C}$ is the control chain (controllers, actuators, etc). The various inputs to the system are then $\mathbf{o}_{\text {rn }}$, the vector of read-out noises for each output channel; $\mathbf{o}_{i}$, any guidance inputs to the system; and $\mathbf{g}$, any force inputs to the system, either deliberate force per unit mass inputs or noise sources. Figure 3 shows a schematic representation of the model described by equation (1).

To recover the residual force per unit mass of the SC and the TMs, we can invert this system equation and rearrange it to obtain

$$
\mathbf{g}=\mathbf{D}^{-1} \mathbf{S}^{-\mathbf{1}}\left(\mathbf{o}-\mathbf{o}_{\mathrm{rn}}\right)+\mathbf{C}\left(\mathbf{o}-\mathbf{o}_{\mathrm{i}}\right)
$$

which can be read as follows. Starting from the system outputs, we invert the sensing and the dynamics of the system, and then (because we have a closed-loop system) we account for the feedback to recover the out-of-loop (or residual) forces.

Each of the terms $\mathbf{D}, \mathbf{S}$ and $\mathbf{C}$ are, in practice, decomposed into further subsystems, each of which contains parameters. Some of these parameters are known by construction, some of them can be determined once and for all through dedicated measurements, and some of them are unknown and variable, depending on the state of system. This last category of parameters must be identified through a series of experiments designed for the purpose. These experiments will typically be repeated under different conditions throughout mission operations.

Once the system is characterized we can compute the residual acceleration of the testmasses by filtering the data according to the time-discrete versions of the equations above. 
When accounting for the commanded forces, we can either use the telemetered signals (if downlink bandwidth permits) or compute them using digital filters which represent the control chain. Further details of the process for recovering the residual force per unit mass of the SC and TMs can be found in [2].

So far we have only mentioned experiments which allow the characterization of the system response. Other experiments are needed if we are to build up a full description of the observed noise. For example, we will certainly need to perform experiments to investigate the thermal and magnetic couplings in the system, the couplings between different degrees of freedom, as well as the performance and coupling of the different noise sources in the optical metrology subsystem. Full details of each of these experiments are out of the scope of this paper, but further discussion can be found in [7]. In the following section, we focus on one of the main experiments: system identification along the $x$-axis.

\subsection{System identification along the $x$-axis}

As already stated above, one of the key steps in building up an accurate physical model of the LPF is the characterization of the dynamical behaviour of the full system along the most sensitive axis, the $x$-axis. It is important to note that the dynamical time constants involved in the system are of the same scale of the length of an individual experiment (around 1 day). This means that we are analysing a system that is never in a steady state. As a result, we try, whenever possible, to use time-domain approaches to ensure that the transient behaviour of the system is properly accounted for.

The characterization of the dynamical system along the $x$-axis is done using a model which contains a number of physical parameters. Each of these parameters must be identified, either on ground or in flight.

When operating in the main science mode, the residual motion of the bodies is minimized due to the closed-loop system. As such, if we treat the system close to this operating point, we can produce a linear parameterization of the components of the system equation shown above (equation (1)) in the following way. First, the dynamics of the system can be described by the following $2 \times 2$ Laplace-domain matrix:

$$
\mathbf{D}^{-1}=\left[\begin{array}{cc}
s^{2}+\omega_{1}^{2}+\frac{m_{1}}{m_{\mathrm{SC}}} \omega_{1}^{2}+\frac{m_{2}}{m_{\mathrm{SC}}} \omega_{2}^{2} & \frac{m_{2}}{m_{\mathrm{SC}}} \omega_{2}^{2}+\Gamma_{x} \\
\omega_{2}^{2}-\omega_{1}^{2} & s^{2}+\omega_{2}^{2}-2 \Gamma_{x}
\end{array}\right]
$$

where $m_{1}, m_{2}$ and $m_{\mathrm{sc}}$ are the masses of the TMs and SC, respectively, the total stiffness coupling of each TM to the SC is represented by the parameters $\omega_{1}^{2}$ and $\omega_{2}^{2}$, and $\Gamma_{x}$ represents the gravitational coupling of the two test-masses. The sensing matrix $\mathbf{S}$ can be represented by

$$
\mathbf{S}=\left[\begin{array}{ll}
S_{11} & S_{12} \\
S_{21} & S_{22}
\end{array}\right]
$$

The control chain can be modelled as known controller functions together with an unknown input delay due to the transmission of the signals from the interferometer/DMU ${ }^{25}$ output along the SC bus to the computer running the control logic. In matrix form, this looks like

$$
\mathbf{C}=\left[\begin{array}{cc}
C_{\text {df }} \mathrm{e}^{-s D_{1}} & 0 \\
0 & C_{\text {sus }} \\
\mathrm{e}^{-s D_{12}}
\end{array}\right]
$$
25 The Data Management Unit (DMU) processes the signals from the interferometer and passes them to the onboard
computer for use in the control loops and for telemetering to ground. 
Table 1. The main physical parameters included in our model of the dynamical system of the LPF along the $x$-axis.

\begin{tabular}{|c|c|c|}
\hline Parameter & Typical value & Description \\
\hline$A_{\mathrm{df}}$ & 1.0 & The unknown gain of the thrusters \\
\hline$A_{\text {sus }}$ & 1.0 & The unknown gain of the electrostatic actuators \\
\hline$S_{11}$ & 1.0 & $\begin{array}{l}\text { The calibration of the interferometer which } \\
\text { senses the } x \text {-axis position of the SC relative to first TM }\left(X_{1}\right)\end{array}$ \\
\hline$S_{12}$ & 0.0 & $\begin{array}{l}\text { The cross-contamination from the } X_{12} \text { interferometer to the } \\
X_{1} \text { interferometer. This represents the sensing of the motion of } \\
\text { TM2 by the } X_{1} \text { interferometer; it is expected to be negligible }\end{array}$ \\
\hline$S_{21}$ & $1 \times 10^{-4}$ & $\begin{array}{l}\text { The cross-contamination from the } X_{1} \text { interferometer to the } X_{12} \\
\text { interferometer. This represents the imperfect common-mode } \\
\text { rejection of the differential interferometer }\end{array}$ \\
\hline$S_{22}$ & 1.0 & The calibration of the differential interferometer $\left(X_{12}\right)$ \\
\hline$\omega_{1}^{2}$ & $-1 \times 10^{-6} \mathrm{~s}^{-2}$ & The total stiffness of TM 1 to the SC \\
\hline$\omega_{\Delta}^{2}$ & $-1 \times 10^{-6} \mathrm{~s}^{-2}$ & $\begin{array}{l}\text { The difference of stiffness of the two TMs to the SC, } \\
\text { defined as } \omega_{2}^{2}-\omega_{1}^{2}\end{array}$ \\
\hline$\tau_{\text {thrust }}$ & $0.1 \mathrm{~s}$ & The characteristic time of the thrusters \\
\hline$\tau_{\text {electro }}$ & $0.01 \mathrm{~s}$ & The characteristic time of the electrostatic actuators \\
\hline$D_{1}$ & $0.1 \mathrm{~s}$ & $\begin{array}{l}\text { The bus delay between the } X_{1} \text { interferometer output } \\
\text { and the corresponding DFACS controller input }\end{array}$ \\
\hline$D_{12}$ & $0.1 \mathrm{~s}$ & $\begin{array}{l}\text { The bus delay between the } X_{12} \text { interferometer output } \\
\text { and the corresponding DFACS controller input }\end{array}$ \\
\hline
\end{tabular}

Finally, the actuator is modelled with an unknown gain and a response time is characterized by a single pole, as follows:

$$
\mathbf{A}=\left[\begin{array}{cc}
\frac{A_{\mathrm{df}}}{1+s \tau_{\text {thrust }}} & 0 \\
0 & \frac{A_{\text {sus }}}{1+s \tau_{\text {electro }}}
\end{array}\right]
$$

Table 1 lists the parameters that our model contains together with the predicted values. In the following section, a pair of experiments is proposed that should lead to the identification of the parameters given in table 1 .

3.1.1. The proposed $x$-axis experiments. If we focus only on the $x$-axis of the system, then we see that we have five inputs at our disposal where we can inject stimulus signals.

(i) The set-point of the drag-free loop can be sinusoidally modulated at a chosen frequency, amplitude and phase, for a given duration. This is a so-called guidance input. This results in the SC motion at the modulation frequency, with a relative phase and amplitude determined by the closed-loop control function. Residual couplings (for example, differences in the overall stiffness of the two TMs) will also result in a parasitic signal in the $X_{12}$ interferometer, and as such in motion of TM2.

(ii) The set-point of the electrostatic suspension control loop which maintains the distance between the two TMs can be similarly modulated. Again, this is a guidance input. This will result in the position of TM2 being modulated. 
(iii) We can command additional forces to the two TMs via the electrostatic actuators. Again, we can apply sinusoidally modulated forces as above.

(iv) Finally, we can command a sinusoidally modulated force to the SC via the micro-Newton thrusters.

For these five inputs, there are four telemetry signals which make sense to look at:

(i) The output of the $X_{1}$ interferometer. We refer to this signal as $o_{1}(t)$.

(ii) The output of the $X_{12}$ interferometer. We refer to this signal as $o_{12}(t)$.

(iii) The control force that will be applied electrostatically to the second TM. We refer to this signal as $F_{\mathrm{TM} 2}(t)$.

(iv) The control force that will be applied to the SC via the micro-Newton thrusters. We refer to this signal as $F_{\mathrm{SC}}(t) .{ }^{26}$

Given these five inputs and four outputs, various experiments have been designed to probe the physical parameters we want to assess. Full details of the experiments are given in [10]. The first two experiments that have been designed involve the injection of guidance signals in to each of the two longitudinal ( $x$-axis) loops. The first experiment is to inject a sequence of sinusoids in to the drag-free loop guidance input; the second experiment involves the injection of a similar sequence of sinusoids in to the suspension loop guidance input. The frequencies and durations of the individual sinusoidal signals are chosen so that we sample the full measurement band in about $10000 \mathrm{~s}$. The amplitudes of the signals are made as large as possible, given the dynamic range of the actuators. In section 4.3 , these experiments are simulated and an analysis of the data is presented in section 5.1.

\section{Simulations and modelling}

Establishing of a full noise budget for LTP requires the development of detailed parametric models which can be used to generate outputs of the system for given inputs. These models are used in two primary ways: to simulate the outputs of the system for given parameter values and noise or signal inputs; and to generate template output signals which can be compared to measured outputs in order to determine different parameters of the system.

Currently, models of LTP have to be generated in various ways, each representing different degrees of complexity and flexibility. We have simple one-dimensional parametric models of the system based on analytic formulae which represent the main characteristics of the system as we expect it to behave along the sensitive $x$-axis. These analytical models have the advantage that they can be directly related to the equations of motion of the system and as such provide a great deal of insight. They are typically Laplace-domain models which we then use to both generate simulated data streams [11] as well as to generate signal templates when performing system identification. These simple 1D models are then assembled in to matrix structures to allow us to handle the multi-input, multi-output (MIMO) nature of the system more effectively.

In addition to the simple analytical models, we have also developed an object-oriented modelling infrastructure within the LISA Technology Package Data Analysis toolbox (LTPDA) which uses a state-space representation of systems to allow us to generate templates and to do time-domain simulations. These state-space models (SSM) are fully three dimensional and contain a higher degree of complexity. Each of the main subsystems in the LPF is modelled individually. These subsystem models can then be assembled to produce a model which

${ }^{26}$ Both this control force and the one commanded to TM2 are not strictly required since they can be reconstructed on ground from knowledge of the control laws and the input signals. 
represents the full closed-loop LPF system in 3D. Since the models also contain any expected coupling paths between the different degrees of freedom, we are able to use the fully assembled system model to assess the impact on the $x$-axis of forces acting along, for example, the $y$-axis. More details of the state-space modelling system can be found in [12].

Finally, we also make extensive use of the mission simulator which was designed by industry [13] for testing and validating the drag-free control system and has now been extended to act as a performance simulator that we can use to validate the planned experiments at the tele-command level. From this simulator, we obtain telemetry data which, at the moment, are the best approximation to the data we expect to obtain from the LPF. As such, it provides an excellent opportunity to test the experiments and analyses on data that were not produced fully under the control of the data analysis team.

\subsection{Noise analysis of models}

The mission simulator contains detailed physical models which were developed by the various architects and industrial companies involved in the construction of the LPF and LTP. As such, we take the mission simulator as a baseline, and validate our models against its outputs. One way of doing that is to attempt to explain the output of the simulator using projections of the various noise disturbances. For example, using the LTPDA 3D state-space model of the LPF we can inject a single noise source (thruster noise, say) and then record the output of the differential interferometer.

Once we have the outputs containing the individual noise contributions, we can then proceed to compute the equivalent residual (out-of-loop) differential forces. To do that, we need to account for the dynamical behaviour of the system, the commanded forces produced due to the control loop, and the additional noise due to the jitter of the SC that contaminates the differential measurement through two coupling paths: via the imperfect common-mode rejection of the interferometer; and via the difference of the spring-like couplings (stiffness) of the two test-masses to the SC. This procedure is detailed in [2].

As an example, the mission simulator is used to generate $72 \mathrm{~h}$ of data. We then take our state-space LPF model together with each of our modelled noise sources in turn, and generate the simulated output of the interferometers for that single noise input. After computing the equivalent differential force-per-unit-mass of each noise contribution, we can then compare the noise spectral density of each of these noise sources to the output of the mission simulator. In addition, we sum together all of these noise sources and look at the spectral density of the correlated sum. The results are presented in figure 4.

\subsection{Simulating experiments}

In the earlier stages of developing the data analysis for the mission, simulating the experiments was done under the framework of Mock Data Challenges (see [9, 14] for details). The main focus at that time was the development of the core algorithms needed to perform the different analyses and the data was all generated using LTPDA noise generators. As such the models used for generating and analysing the data were typically one and the same.

Recently, the focus has shifted to the validation of the experiments themselves. This means that we first need to confirm that the experiment as planned (typically in supporting technical notes) can actually be performed given the hardware and tele-command options at our disposal. The experiments are quite diverse in scope, covering system identification, noise budget analysis, residual acceleration determination [2], analysis and projections of 


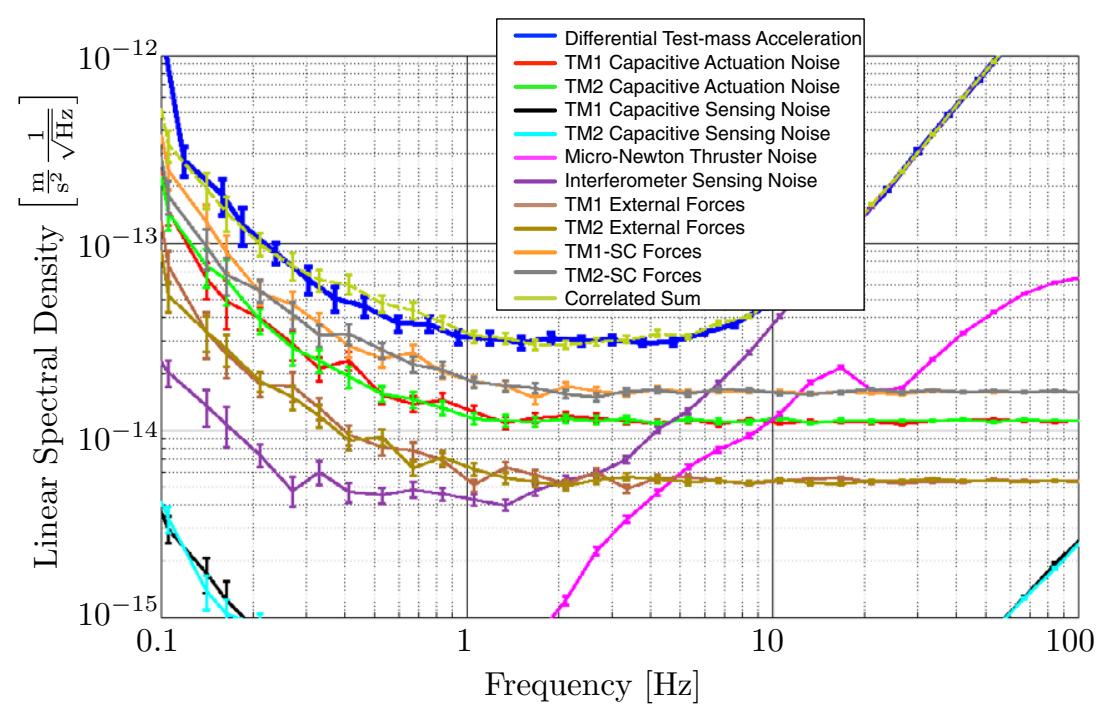

Figure 4. A linear projection of the noise sources to differential force-per-unit-mass of the TMs. The thick upper (blue) trace represents the data from the mission simulator whereas the total correlated sum of the other noise sources is shown by the dashed (yellow) trace. Each spectrum is computed from the original time-series by using the Welch overlap method with 16 segments. The resulting spectrum is then binned in frequency with 10 points per decade.

environmental effects [15-17], as well as more specialized experiments like the free-fight experiment [18].

Often the planned experiments need slight adjustment to fit the constraints of the system. Having designed the tele-command sequence needed to run the experiment, we are able to use the mission simulator to produce data based on that tele-command sequence, giving us a data set based on a model of the system that is independent from the models we will use to characterize the data; this scenario is much closer to the conditions we expect to face during the mission.

The validation of the experiments and their associated analysis pipelines is now performed under a framework of operation exercises, where we not only test the analysis tools and pipelines, but also our ability to carry out the analysis under time constraints similar to those we will face during the mission. Each operational exercise focusses on a particular experiment. The tele-command sequence (typically covering one day of mission time) is prepared in advance and the data are generated using the mission simulator. The exercise then begins with the retrieval of the simulated data from the LTPDA repository. The aim is to perform a front-line analysis of the data in one day. The following section describes an example of one of these operational exercises and section 5.1 presents the preliminary results obtained during the exercise.

\subsection{Example: simulating guidance injections in the drag-free loop}

The experiments described in section 3.1.1 can be simulated using the mission simulator. The simulation starts with the LTP in 'accelerometer mode'. That is, the position of both TMs relative to the SC is controlled locally via the electrostatic sensing and actuation. After some time, the system is placed in Science Mode 1 (SM1) where the first TM is drag-free and the 
$\mathrm{SC}$ is made to follow by sensing the relative position to the drag-free TM via the capacitive sensor. In this mode, the position of the second TM relative to the drag-free TM is read out using the differential interferometer and maintained via electrostatic actuation. After some further time, the system switches to Science Mode 1 all-optical. Here the only change is that the position of the SC relative to the drag-free test-mass is interferometrically read out.

The system is then allowed to settle and a few hours of 'noise' data are collected. Towards the end of the simulation, a series of sinusoidal signals are injected as guidance inputs to the drag-free control loop. The strategy chosen is to excite the system at different frequencies across the measurement band (from around $1 \mathrm{mHz}$ to around $50 \mathrm{mHz}$ ) in order to allow some of the parameters to be discriminated by their frequency-dependent effect.

Figure 5 shows the simulated outputs of the interferometer channels, $o_{1}(t)$ and $o_{12}(t)$, with the different phases of the simulation indicated. If we observe the response of the system to these input signals by looking at the data of the two interferometer outputs $\left(o_{1}\right.$ and $\left.o_{12}\right)$, then we obtain information about:

- the thruster actuation gain $\left(A_{\mathrm{df}}\right)$.

- the electrostatic actuator gain ( $\left.A_{\text {sus }}\right)$. Here the effect is to influence the closed-loop response of the suspension loop, and hence the amount of signal leaked in to the suspension loop via the two cross-couplings described below since both cross-coupling paths result in signal being added in to the loop.

- the total stiffness of the drag-free TM to the SC $\left(\omega_{1}^{2}\right)$. The effect of this parameter is to change the loop gain at low frequencies (around $1 \mathrm{mHz}$ ) and thus is observable in the comparison of $o_{1}$ to the injected signals at the lowest injection frequencies.

- the difference of the total stiffness of the two TMs to the SC $\left(\omega_{\Delta}^{2}=\omega_{2}^{2}-\omega_{1}^{2}\right)$. This is primarily identified in the cross-contamination of the two control loops at low frequencies. The actual physical coupling arises from motion of the SC coupling differently to the two TMs and hence resulting in a change in their differential position.

- the imperfect common-mode rejection of the differential interferometer $\left(S_{21}\right)$ which causes a coupling between the two control loops at all frequencies.

The remaining parameters associated with the delays in the system ( $\tau_{\text {thrust }}, \tau_{\text {electro }}, D_{1}$, and $D_{12}$ ) affect the closed-loop response of the two control loops in a way similar to the actuator gains described above, except that they only play a significant role at the higher frequencies, and as such can be separated in effect from the other parameters. Also, we should note that the two main cross-coupling effects (from the difference of stiffness, $\omega_{\Delta}^{2}$, and from the imperfect common-mode rejection of the interferometer, $S_{21}$ ) should be separable in frequency since the stiffness coupling is frequency dependent and falls off towards high frequency where the interferometer coupling takes over.

\section{Analysis of the experiments}

All the planned experiments require drawing on various data analysis and signal processing techniques, for example, spectral estimation, system identification, transfer function estimation, and digital filtering. Many of the experiments we will carry out are devoted to the identification of the various parameters of the system. The analysis of those particular experiments needs to extract estimations of the parameters. Typically we have a MIMO system model, and we perform a series of experiments devoted to identifying a particular set of parameters. This set of experiments then needs to be analysed together to properly determine correlations in the parameters and to ensure the best possible precision for each parameter estimate. 

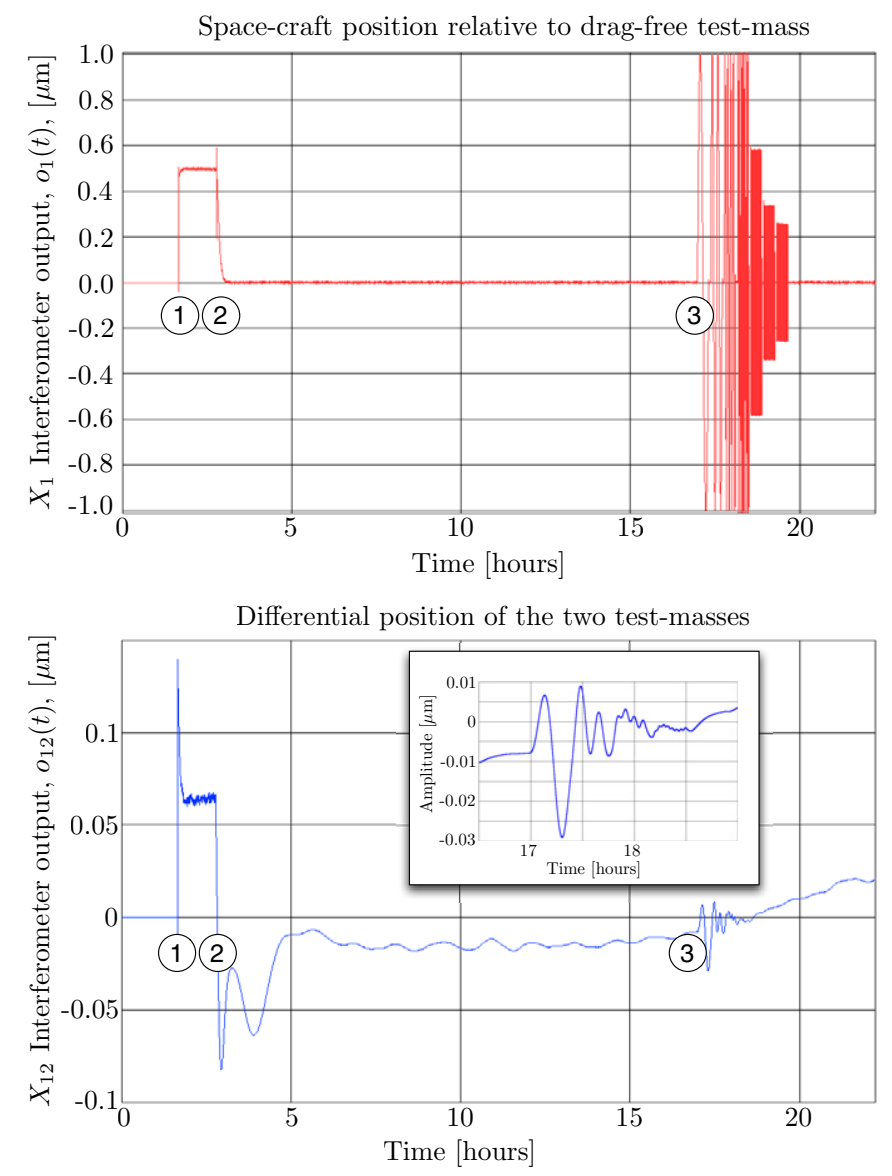

Figure 5. The simulation outputs of the two interferometer channels when modulating the set-point of the drag-free loop. At the start of the simulation, the LPF is in accelerometer mode and the interferometer is switched off. After about $1.5 \mathrm{~h}$ the system goes to Science Mode 1 which includes activating the interferometer (marker 1). After another hour elapses, the system goes to Science Mode 1 all-optical and we see that the position of the SC is now controlled on the interferometer output, hence it is centred on zero (marker 2). After collecting some hours of noise data, the series of sinusoidal injections begins at about $T=17 \mathrm{~h}$ (marker 3 ).

Given a model of the system, we can produce template outputs for a given set of parameter values and input signals, and compare these to the measured outputs. The parameters of the system are then adjusted to minimize the difference between the predicted outputs and the measured/observed outputs. This minimization is done using a variety of methods, for example, Markov chain Monte Carlo (mcmc) [19, 20], nonlinear least-squares [21], and linear least-squares [21].

Since the mission timeline will depend on the results of earlier experiments, some of these parameters need to be estimated on-line. Therefore, for operations, we focus on the simplest, fastest and most robust approach that can get us the information we require. Currently, this is expected to be a modified linear least-squares approach (see section 5.1). This method is particularly appropriate since we will typically have good starting estimates 
for the various system parameters, so convergence to a global minimum is expected. In addition to this quasi-real-time analysis, more in-depth analyses will be performed in parallel but without the time constraint. In this off-line data analysis, more sophisticated techniques will be employed to recover the best possible estimates of the system parameters given the data we will have. During the development phase, we aim to validate the different tools against each other. In the following section, we describe in more detail, the modified linear least-squares fitting approach that we intend to use for the on-line analysis during operations.

\subsection{Parameter estimation via linear least-squares fitting}

As stated earlier, given that we will have reasonably accurate starting values for many of the parameters we are trying to identify, an obvious way to determine the parameter values is via a standard linear least-squares fitting algorithm.

Since we deal with finite length time series, then the response of the system, $\mathbf{G}$, to a given input, $\mathbf{o}_{\mathbf{i}}(n)$, can be represented in the frequency domain as

$$
\mathbf{o}(\Omega)=\mathbf{G}(\Omega) \mathbf{o}_{\mathbf{i}}(\Omega),
$$

where $\Omega$ is the normalized angular frequency defined as $\Omega=2 \pi f / f_{\mathrm{s}}$, where $f_{\mathrm{s}}$ is the sampling frequency of the data. As the time series is of finite duration, $\Omega$ can assume only finite values $\Omega=\frac{2 \pi k}{N}, k=0, \ldots, \frac{N}{2}$. The interferometer output vector is labelled $\mathbf{o}$, and $\mathbf{0}_{\mathbf{i}}$ is the guidance input vector on the drag-free and electrostatic suspension control loops. $\mathbf{G}(\Omega)$ depends not only on the frequencies, $\Omega$, but also on the dynamical parameters, i.e. $\mathbf{G} \rightarrow \mathbf{G}\left(\Omega, p_{1}, \ldots, p_{M}\right)$. Equation (8) can be Taylor-expanded to the first order in terms of the parameters

$$
\begin{gathered}
o(\Omega) \approx \mathbf{G}\left(\Omega, p_{1_{0}}, \ldots, p_{M_{0}}\right) o_{i}(\Omega)+\cdots \\
\sum_{i=1}^{M} \delta p_{i}\left[\frac{\partial \mathbf{G}\left(\Omega, p_{1}, \ldots, p_{M}\right)}{\partial p_{i}}\right]_{p_{i} \rightarrow p_{i_{0}}} o_{i}(\Omega) .
\end{gathered}
$$

The term $\mathbf{G}\left(\Omega, p_{1_{0}}, \ldots, p_{M_{0}}\right) o_{i}(\Omega)$ is the so-called nominal response of the system, i.e.the response of the system computed with the initial values of the dynamical parameters, $p_{1_{0}}, \ldots, p_{M_{0}}$. The second term on the right-hand side of the equal sign is the linear term which contains the unknown parameters $\delta p_{i}, i=1, \ldots, M$. We will refer to the terms $\left[\frac{\partial \mathbf{G}\left(\Omega, p_{1}, \ldots, p_{M}\right)}{\partial p_{i}}\right]_{p_{i} \rightarrow p_{i_{0}}} o_{i}(\Omega)$ as the fit basis.

Since the system model is represented in the frequency domain and the data series are in time domain, the linear fit operation corresponds to the solution of the system of coupled equations:

$$
\begin{aligned}
& o(n)-\mathcal{F}^{-1}\left\{\mathbf{G}\left(\Omega, p_{1_{0}}, \ldots, p_{M_{0}}\right) o_{i}(\Omega)\right\}=\cdots \\
& \quad \sum_{i=1}^{M} \delta p_{i} \mathcal{F}^{-1}\left\{\left[\frac{\partial \mathbf{G}\left(\Omega, p_{1}, \ldots, p_{M}\right)}{\partial p_{i}}\right]_{p_{i} \rightarrow p_{i_{0}}} o_{i}(\Omega)\right\} \\
& n=0, \ldots, N-1 .
\end{aligned}
$$

We indicated the inverse Fourier transform with $\mathcal{F}^{-1}$ and applied its linearity properties. Once the system of equations is solved, the final estimation for the dynamical parameters will be $p_{i}=p_{i_{0}}+\delta p_{i}$ for $i=1, \ldots, M$. The procedure is iterated over in order to arrive at a minimum least-squares solution and can be summarized by the following steps: 
(i) Whiten data. The noise on our data is non-white, and the individual outputs are essentially uncorrelated since the cross-coherence between the two output channels, given the typical values of the cross-coupling terms, is consistent with zero. In order to estimate the uncertainties on the recovered parameter values, we employ standard textbook methods ${ }^{27}$ applicable when doing a linear least-squares fit, which then forces us to whiten the data with a whitening filter before the fitting operation.

(ii) Basis change. The fit basis is composed of linearly dependent elements since several parameters are correlated. The system cannot be solved under such conditions; therefore, we perform a change of basis by using the singular value decomposition (SVD) algorithm [21]. The SVD ensures that we deal with a linearly independent fit basis.

(iii) Generate whitened templates. In order to correctly perform the fit, both the nominal response templates and the fit basis templates need to be whitened using the same whitening filter described above.

(iv) Fit. The system of normal equations is solved using standard techniques in order to get an estimate of the parameters.

(v) Check convergence and go to (iii). The process is iterated until the convergence of the parameters is reached. At each step the nominal value of each of the parameters is updated with the current knowledge. This helps reduce any bias that our linear estimator gains due to the initial parameter values. If we call $p_{i_{\Delta}}$ the change in the parameter $p_{i}$ from one iteration to the next, and $\sigma_{p_{i}}$ the estimated error on $p_{i}$, then convergence is deemed to be achieved when the ratio $\sigma_{p_{i}} / p_{i_{\Delta}}$ falls below a user-settable threshold (here we took 1 part in $\left.10^{5}\right)$.

(vi) Change basis back to physical parameters. At this stage, we collect the parameter estimates from different channels, experiments and on-ground measurements and apply the conversion from the orthogonal fit parameters back to the desired physical parameters. (Essentially, we undo the SVD applied in step (ii).)

\subsection{Application to $x$-axis system identification}

As an example, this procedure was applied to the two simulated experiments described in section 4.3. The simulations were run with a sampling frequency of $10 \mathrm{~Hz}$. In this case, we fit the parameters listed in table 2; the recovered parameter estimates are also given in that table. The remaining system parameters $\left(S_{11}, S_{12}\right.$, and $\left.S_{22}\right)$ were given values as if they were measured on ground.

Having performed the fit, we can go on to compare the residuals after subtracting the (fitted) template waveform from the data. Figure 6 shows the power spectral density of the residuals of the whitened output of the $X_{12}$ interferometer from the first guidance injection experiment (see section 3.1.1) before and after the fit procedure. We see that the residuals after the fit are consistent with white noise, as expected. It is difficult to directly compare the parameter values we estimated during the fitting process to the 'true' values present in the simulator, since the model used in the data analysis, and the model used in the simulator are parameterized differently. However, some of the parameters can be compared. For example, the estimations of the stiffness of the two test-masses were in agreement with those present in the simulator.

${ }^{27}$ To estimate the uncertainties, the Jacobian, $J$, of the $\chi^{2}$ function is computed and then used to estimate the Hessian as $H=J^{\mathrm{T}} J$, which is then inverted to give an estimation of the covariance matrix. See chapter 15 of [21] for more details. 


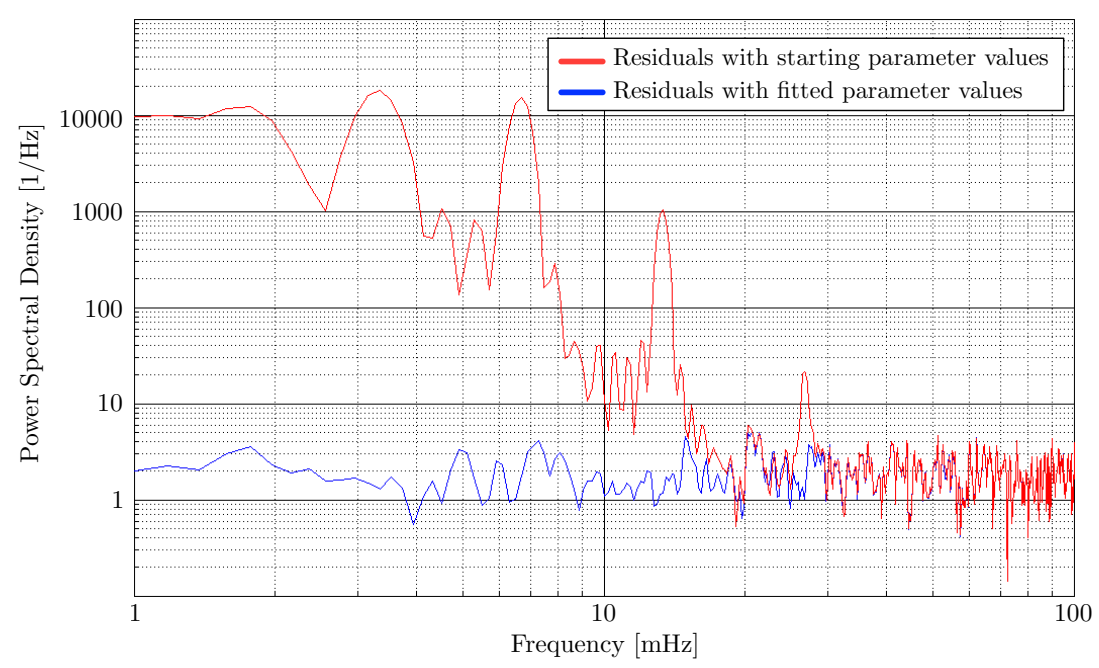

Figure 6. The spectrum of the whitened residuals for the differential TM position measured during the first guidance injection experiment described in section 3.1.1. The upper (red) trace shows the residuals using the starting parameter values; the lower (blue) trace shows the residuals after performing the fit procedure. The spectra are computed using a single window on the data, i.e.no averaging is performed.

Table 2. The parameter values obtained from linear fit analysis of the two $x$-axis system identification experiments described in section 3.1.1. The last column gives an estimate of the expected errors obtained from the inverse of a Fisher matrix calculation. The true, underlying parameters of the system are not available for comparison since we approximate the system using our linear model. As such, the quality of the fit has to be judged by inspection of the residuals.

\begin{tabular}{llll}
\hline & \multicolumn{2}{c}{ Linear fit results } & \multirow{2}{*}{ Expected errors } \\
\cline { 2 - 3 } Parameter & Value & $\sigma$ & $\sigma$ \\
\hline$A_{\mathrm{df}}$ & 1.08 & 0.0005 & 0.0004 \\
$A_{\text {sus }}$ & 1.0 & $6 \times 10^{-5}$ & $2.2 \times 10^{-5}$ \\
$S_{21}$ & $1.1 \times 10^{-6}$ & $4 \times 10^{-7}$ & $3 \times 10^{-7}$ \\
$\omega_{1}^{2}\left(\mathrm{~s}^{-2}\right)$ & $-1.32 \times 10^{-6}$ & $4 \times 10^{-9}$ & $1.4 \times 10^{-9}$ \\
$\omega_{\Delta}^{2}\left(\mathrm{~s}^{-2}\right)$ & $-7.16 \times 10^{-7}$ & $6 \times 10^{-10}$ & $4.7 \times 10^{-10}$ \\
$\tau_{\text {thrust }}(\mathrm{s})$ & 0.417 & 0.002 & 0.001 \\
$\tau_{\text {electro }}(\mathrm{s})$ & 0.201 & 0.003 & 0.001 \\
$D_{1}(\mathrm{~s})$ & 0.197 & 0.0003 & 0.0002 \\
$D_{12}(\mathrm{~s})$ & 0.2 & 0.009 & 0.003 \\
\hline
\end{tabular}

\section{Concluding remarks}

The development of the data analysis activities to be performed during the operational phase of LISA Pathfinder covers many aspects. These include infrastructure construction; the preparation and building of data analysis tools; the design, testing and validation of experiments; the design, testing and validation of the corresponding data analysis pipelines; and, last, but not least, the forming and training of a team able to carry out the data analysis during the mission operations. 
Many of these aspects have been touched upon in this paper, and in some cases further references are given to allow the reader to find more detailed information. In summary, the various pieces of the data analysis chain are all well on track to be ready for the launch of LISA Pathfinder in 2013. In the intervening period, the majority of the effort will be on the design and optimization of the experiments we plan to perform. In addition, the data from simulated experiments will be analysed to validate the analysis algorithms and tools. By being as prepared as possible, and by having a robust and flexible data analysis environment available to us, we fully expect to extract the maximum science from the LPF data.

The characterization of LISA Pathfinder will provide invaluable information for the development and construction of LISA, in particular the ability to project the LISA Pathfinder noise budget forward will help guide the development of the LISA hardware, particularly for those subsystems where we expect to be able to reuse LPF technology. In addition, the study of the couplings and noise sources in such a complex dynamical system will allow us to improve the simulation of the LISA data that are heavily used in the development of the data analysis algorithms needed for the extraction of astrophysical information from the LISA data. Some effort in this direction has already started (see [22]), and will likely increase when the data from LISA Pathfinder is analysed.

\section{References}

[1] Bender P et al 2000 LISA: a cornerstone mission for the observation of gravitational waves System and Technology Study Report ESA-SCI (2000)11

[2]Ferraioli L et al 2011 Analysis of the residual force noise for the LISA Technology Package Proc. 8th Int. LISA Symp., (LISA 8) (Stanford University, California, USA, 28 June-2 July 2010)

[3] Fichter W, Gath P, Vitale S and Bortoluzzi D 2005 Class. Quantum Grav. 22 139-48

[4] Gerndt R and the entire LTP Team 2009 J. Phys.: Conf. Ser. 154012007

[5] Dolesi R et al 2003 Class. Quantum Grav. 20 S99

[6] Steier F, Cervantes F G, Marin A F G, Gerardi D, Heinzel G and Danzmann K 2009 Class. Quantum Grav. 26094010

[7] Antonucci F et al 2011 From laboratory experiments to LISA Pathfinder: achieving LISA geodesic motion Class. Quantum Grav. 28094002

[8] Bortoluzzi D, Carbone L, Cavalleri A, Lio M D, Dolesi R, Hoyle C D, Hueller M, Vitale S and Weber W J 2004 Class. Quantum Grav. 21 S573

[9] Hewitson M et al 2009 Class. Quantum Grav. 26094003

[10] Vitale S 2008 Measurement of LTP dynamical coefficients by system identification Technical Report S2-UTNTN-3045 Issue 2, University of Trento

[11] Ferraioli L, Hueller M, Vitale S, Heinzel G, Hewitson M, Monsky A and Nofrarias M 2010 Phys. Rev. D 82042001

[12] Aguilo M D et al 2011 Modeling LISA pathfinder for data analysis Talk presented at Proc. 8th Int. LISA Symp. (LISA 8) (Stanford University, California, USA, 28 June-2 July 2010)

[13] Brandt N et al 2004 Autom. Remote Control 66 241-6

[14] Monsky A et al 2009 Class. Quantum Grav. 26094004

[15] Lobo A et al 2006 AIP Conf. Proc. 841 489-92

[16] Lobo A, Nofrarias M, Ramos-Castro J and Sanjuan J 2006 Class. Quantum Grav. 23 5177-94

[17] Nofrarias M et al 2009 J. Phys.: Conf. Ser. 154012004

[18] Grynagier A et al 2010 Proc. 8th Int. LISA Symp. J. Phys.: Conf. Ser.

[19] Gelman A, Carlin J B, Stern H and Rubin D B 1997 Bayesian Data Analysis (London: Chapman and Hall/CRC Press)

[20] Gilks W R, Richardson S and Spiegelhalter D J 1996 Markov Chain Monte Carlo in Practice (London: Chapman and Hall/CRC Press)

[21] Press W H, Teukolsky S A, Vetterling W T and Flannery B P 2007 Numerical Recipes: The Art of Scientific Computing 3rd edn (New York: Cambridge University Press)

[22] Monsky A, Hewitson M, Petiteau A, Plagnol E, Heinzel G and Danzmann K 2011 Injecting a non-Gaussian noise source into LISA Talk presented at Proc. 8th Int. LISA Symp. (LISA 8) (Stanford University, California, USA, 28 June-2 July 2010) 